Supporting Information for Analytical Chemistry

Extractive Solubilization, Structural Change and Functional Conversion of Cytochrome $c$ in Ionic Liquids via Crown Ether Complexation

Kojiro Shimojo, ${ }^{*}{ }^{\dagger}$ Noriho Kamiya, ${ }^{\ddagger}$ Fumito Tani, ${ }^{\S}$ Hirochika Naganawa, ${ }^{\dagger}$ Yoshinori Naruta ${ }^{\S}$ and Masahiro Goto*,"

Division of Environment and Radiation Sciences, Nuclear Science and Engineering Directorate, Japan Atomic Energy Agency, Tokai-mura, Ibaraki, 319-1195, Japan, Department of Applied Chemistry, Graduate School of Engineering and Center for Future Chemistry, Kyushu University, 744 Motooka, Fukuoka, 819-0395, Japan, and Institute for Materials Chemistry and Engineering, Kyusyu University, Higashi-ku, Fukuoka 812-8581, Japan

*To whom correspondence should be addressed. E-mail: shimojo.kojiro@jaea.go.jp (K.S.); mgototcm@mbox.nc.kyushu-u.ac.jp (M.G.)

Phone: +81-29-282-5090 (K.S.); +81-92-802-2806 (M.G.)

Fax: +81-29-282-6757 (K.S.); +81-92-802-2810 (M.G.)

${ }^{\dagger} J a p a n$ Atomic Energy Agency

${ }^{\ddagger}$ Kyushu University

${ }^{\S}$ Kyushu University 


\section{IL Syntheses.}

Syntheses of $\left[\mathrm{C}_{2} \operatorname{mim}\right]\left[\mathrm{Tf}_{2} \mathrm{~N}\right],\left[\mathrm{C}_{\mathrm{n}} \mathrm{OHmim}\right]\left[\mathrm{Tf}{ }_{2} \mathrm{~N}\right](\mathrm{n}=2,3,6,8)$ and $\left[\mathrm{C}_{2} \mathrm{OC}_{1} \operatorname{mim}\right]\left[\mathrm{Tf}_{2} \mathrm{~N}\right]$ were conducted as follows. Under a nitrogen atmosphere, bromoethane (for $\left[\mathrm{C}_{2} \mathrm{mim}\right][\mathrm{Br}]$ ), bromoethanol (for $\left[\mathrm{C}_{2} \mathrm{OHmim}\right][\mathrm{Br}]$ ), 3-bromo-1-propanol (for $\left[\mathrm{C}_{3} \mathrm{OHmim}\right][\mathrm{Br}]$ ), bromoethyl methyl ether (for $\left[\mathrm{C}_{2} \mathrm{OC}_{1}\right.$ mim $][\mathrm{Br}]$ ), 6-chloro-1-hexanol (for $\left[\mathrm{C}_{6} \mathrm{OHmim}\right][\mathrm{Cl}]$ ) or 8-chloro-1octanol (for $\left[\mathrm{C}_{8} \mathrm{OHmim}\right][\mathrm{Cl}]$ ) were carefully added to an equal amount of 1-methylimidazole in an ice bath. The analogous bromide salts were prepared by reaction for $24 \mathrm{~h}$ at room temperature, and the analogous chloride salts were prepared by heating for $72 \mathrm{~h}$ at $70{ }^{\circ} \mathrm{C}$. The resulting solids or viscous liquids were washed with ethyl acetate. After the last washing, the remaining ethyl acetate was removed by heating up to $90{ }^{\circ} \mathrm{C}$ under vacuum and freeze-drying.

The final bis(trifluoromethanesulfonyl)imide type ILs were prepared by slowly adding bistrifluoromethanesulfonimide lithium salt $(1.1 \mathrm{~mol}$ eq. $)$ to the corresponding halide in deionized water. After stirring for $12 \mathrm{~h}$, the upper aqueous layer was decanted and the lower ionic liquid portion was washed with deionized water 10 times to remove any remaining $\mathrm{LiCl}$ or $\mathrm{LiBr}$. The remaining water was removed by heating up to $90{ }^{\circ} \mathrm{C}$ under vacuum and freeze-drying. The ILs were stored in a vacuum desiccator.

Synthesis of $\left[\mathrm{C}_{3} \mathrm{UC}_{4} \operatorname{mim}\right]\left[\mathrm{Tf}_{2} \mathrm{~N}\right]$ was conducted as follows. Under a nitrogen atmosphere, butyl isocyanate $(16.0 \mathrm{~g}, 0.19 \mathrm{~mol})$ was mixed with dry acetonitrile $(25 \mathrm{~mL})$, and the solution was carefully added to 1-(3-aminopropyl)imidazole $(20.0 \mathrm{~g}, 0.16 \mathrm{~mol})$ in dry acetonitrile $(100 \mathrm{~mL})$. The reaction was stirred overnight at room temperature. The unreacted butyl isocyanate and solvent were evaporated under reduced pressure. The resulting liquid was washed with hexane. The product was dissolved in chloroform and washed with deionized water. After the last washing, the remaining solvents were removed under vacuum and freeze-drying. 
Under a nitrogen atmosphere, the precursor $(35.1 \mathrm{~g}, 0.156 \mathrm{~mol})$ was redissolved in dry acetonitrile $(100 \mathrm{~mL})$, and iodomethane $(22.2 \mathrm{~g}, 0.156 \mathrm{~mol})$ was carefully added. The mixture was stirred for $12 \mathrm{~h}$ at room temperature. The solvent was evaporated under reduced pressure, and the resulting liquid was washed with ethyl acetate. After the last washing, the remaining ethyl acetate was removed under vacuum and freeze-drying.

The iodide type IL (50.0 g, $0.137 \mathrm{~mol})$ was dissolved in deionized water, and bistrifluoromethanesulfonimide lithium salt (1.1 mol eq.) was added. After stirring for $12 \mathrm{~h}$, the upper aqueous layer was decanted and the lower ionic liquid portion was washed with deionized water 10 times. The remaining water was removed by heating up to $90{ }^{\circ} \mathrm{C}$ under vacuum and freeze-drying. The ILs were stored in a vacuum desiccator.

$\left[\mathrm{C}_{2} \operatorname{mim}\right]\left[\mathrm{Tf}_{2} \mathrm{~N}\right]: 89.8 \%$ yield; ${ }^{1} \mathrm{H}$ NMR $\left(250 \mathrm{MHz}\right.$, Acetone- $\left.d_{6}, \mathrm{TMS}, 25{ }^{\circ} \mathrm{C}\right) \delta 1.47(\mathrm{t}, 3 \mathrm{H}, \mathrm{N}-$ $\left.\mathrm{CH}_{2}-\mathrm{CH}_{3}\right), 3.85\left(\mathrm{~s}, 3 \mathrm{H}, \mathrm{CH}_{3}-\mathrm{N}\right), 4.10\left(\mathrm{q}, 2 \mathrm{H}, \mathrm{N}-\mathrm{CH}_{2}-\mathrm{CH}_{3}\right), 7.34(\mathrm{~d}, 2 \mathrm{H}, \mathrm{N}-\mathrm{CH}-\mathrm{CH}-\mathrm{N}), 8.43$ (s, $1 \mathrm{H}, \mathrm{N}-\mathrm{CH}-\mathrm{N}$ ); Anal. Calcd for $\mathrm{C}_{8} \mathrm{H}_{11} \mathrm{O}_{4} \mathrm{~N}_{3} \mathrm{~S}_{2} \mathrm{~F}_{6}: \mathrm{C}, 24.55 ; \mathrm{H}, 2.83 ; \mathrm{N}, 10.74$. Found: $\mathrm{C}, 24.46 ; \mathrm{H}$, $2.81 ; \mathrm{N}, 10.99$.

$\left[\mathrm{C}_{2} \mathrm{OHmim}\right]\left[\mathrm{Tf}_{2} \mathrm{~N}\right]: 77.2 \%$ yield; ${ }^{1} \mathrm{H}$ NMR $\left(250 \mathrm{MHz}\right.$, Acetone- $d_{6}$, TMS, $\left.25{ }^{\circ} \mathrm{C}\right) \delta 3.39(\mathrm{~s}, 1 \mathrm{H}$, $\mathrm{OH}), 3.81\left(\mathrm{~m}, 5 \mathrm{H}, \mathrm{CH}_{3}-\mathrm{N}, \mathrm{N}-\mathrm{CH}_{2}\right), 4.18\left(\mathrm{t}, 2 \mathrm{H}, \mathrm{N}-\mathrm{CH}_{2}-\mathrm{CH}_{2}\right), 7.26(\mathrm{~d}, 2 \mathrm{H}, \mathrm{N}-\mathrm{CH}-\mathrm{CH}-\mathrm{N}), 8.46(\mathrm{~s}$, $1 \mathrm{H}, \mathrm{N}-\mathrm{CH}-\mathrm{N}$ ); Anal. Calcd for $\mathrm{C}_{8} \mathrm{H}_{11} \mathrm{O}_{5} \mathrm{~N}_{3} \mathrm{~S}_{2} \mathrm{~F}_{6}: \mathrm{C}, 23.59 ; \mathrm{H}, 2.72 ; \mathrm{N}, 10.32$. Found: $\mathrm{C}, 23.52 ; \mathrm{H}$, $2.77 ; \mathrm{N}, 10.55$.

$\left[\mathrm{C}_{3} \mathrm{OHmim}\right]\left[\mathrm{Tf}_{2} \mathrm{~N}\right]: 73.0 \%$ yield; ${ }^{1} \mathrm{H}$ NMR $\left(250 \mathrm{MHz}\right.$, Acetone- $d_{6}$, TMS, $\left.25^{\circ} \mathrm{C}\right) \delta 2.12(\mathrm{~m}, 2 \mathrm{H}$, $\left.\mathrm{CH}_{2}-\mathrm{CH}_{2} \mathrm{OH}\right), 3.63\left(\mathrm{t}, 2 \mathrm{H}, \mathrm{CH}_{2}-\mathrm{OH}\right), 3.88(\mathrm{br}, 1 \mathrm{H}, \mathrm{OH}), 4.05\left(\mathrm{~s}, 3 \mathrm{H}, \mathrm{CH}_{3}-\mathrm{N}\right), 4.52(\mathrm{t}, 2 \mathrm{H}, \mathrm{N}-$ $\left.\mathrm{CH}_{2}\right), 7.73(\mathrm{~d}, 2 \mathrm{H}, \mathrm{N}-\mathrm{CH}-\mathrm{CH}-\mathrm{N}), 8.96(\mathrm{~s}, 1 \mathrm{H}, \mathrm{N}-\mathrm{CH}-\mathrm{N})$; Anal. Calcd for $\mathrm{C}_{9} \mathrm{H}_{13} \mathrm{O}_{5} \mathrm{~N}_{3} \mathrm{~S}_{2} \mathrm{~F}_{6}: \mathrm{C}$, 25.66; H, 3.11; N, 9.97. Found: C, 25.66; H, 3.08; N, 10.18. 
$\left[\mathrm{C}_{6} \mathrm{OHmim}\right]\left[\mathrm{Tf}_{2} \mathrm{~N}\right]: 83.4 \%$ yield; ${ }^{1} \mathrm{H} \mathrm{NMR}\left(250 \mathrm{MHz}\right.$, Acetone- $\left.d_{6}, \mathrm{TMS}, 25{ }^{\circ} \mathrm{C}\right) \delta 1.43(\mathrm{~m}, 4 \mathrm{H}$, $\left.\mathrm{N}-\mathrm{CH}_{2}-\mathrm{CH}_{2}-\left(\mathrm{CH}_{2}\right)_{2}-\right), 1.48\left(\mathrm{~m}, 2 \mathrm{H}, \mathrm{N}-\left(\mathrm{CH}_{2}\right)_{4}-\mathrm{CH}_{2}-\right), 1.96\left(\mathrm{~m}, 2 \mathrm{H}, \mathrm{N}-\mathrm{CH}_{2}-\mathrm{CH}_{2}{ }^{-}\right), 3.53(\mathrm{~m}, 3 \mathrm{H},-$ $\left.\mathrm{CH}_{2}-\mathrm{OH}\right), 4.04\left(\mathrm{~s}, 3 \mathrm{H}, \mathrm{CH}_{3}-\mathrm{N}\right), 4.35$ (t, 2H, N-CH $), 7.68$ (d, 2H, N-CH-CH-N), 8.98 (s, 1H, N$\mathrm{CH}-\mathrm{N}$ ); Anal. Calcd for $\mathrm{C}_{12} \mathrm{H}_{19} \mathrm{O}_{5} \mathrm{~N}_{3} \mathrm{~S}_{2} \mathrm{~F}_{6}: \mathrm{C}, 31.10 ; \mathrm{H}, 4.13 ; \mathrm{N}, 9.07$. Found: $\mathrm{C}, 31.33 ; \mathrm{H}, 4.14 ; \mathrm{N}$, 8.96.

$\left[\mathrm{C}_{8} \mathrm{OHmim}\right]\left[\mathrm{Tf}_{2} \mathrm{~N}\right]: 82.6 \%$ yield; ${ }^{1} \mathrm{H} \mathrm{NMR}\left(250 \mathrm{MHz}\right.$, Acetone- $\left.d_{6}, \mathrm{TMS}, 25{ }^{\circ} \mathrm{C}\right) \delta 1.34(\mathrm{~m}, 8 \mathrm{H}$, $\left.\mathrm{N}-\mathrm{CH}_{2}-\mathrm{CH}_{2}-\left(\mathrm{CH}_{2}\right)_{4}^{-}\right), 1.48\left(\mathrm{~m}, 2 \mathrm{H}, \mathrm{N}-\left(\mathrm{CH}_{2}\right)_{6}-\mathrm{CH}_{2}-\right), 1.94\left(\mathrm{~m}, 2 \mathrm{H}, \mathrm{N}-\mathrm{CH}_{2}-\mathrm{CH}_{2}-\right), 3.51(\mathrm{~m}, 3 \mathrm{H},-$ $\left.\mathrm{CH}_{2}-\mathrm{OH}\right), 4.04\left(\mathrm{~s}, 3 \mathrm{H}, \mathrm{CH}_{3}-\mathrm{N}\right), 4.34\left(\mathrm{t}, 2 \mathrm{H}, \mathrm{N}-\mathrm{CH}_{2}\right), 7.68$ (d, 2H, N-CH-CH-N), 9.08 (s, 1H, N$\mathrm{CH}-\mathrm{N}$ ); Anal. Calcd for $\mathrm{C}_{14} \mathrm{H}_{23} \mathrm{O}_{5} \mathrm{~N}_{3} \mathrm{~S}_{2} \mathrm{~F}_{6}: \mathrm{C}, 34.21 ; \mathrm{H}, 4.72 ; \mathrm{N}, 8.55$. Found: $\mathrm{C}, 34.21 ; \mathrm{H}, 4.74 ; \mathrm{N}$, 8.61 .

$\left[\mathrm{C}_{2} \mathrm{OC}_{1} \operatorname{mim}\right]\left[\mathrm{Tf}_{2} \mathrm{~N}\right]: 73.8 \%$ yield; ${ }^{1} \mathrm{H}$ NMR $\left(250 \mathrm{MHz}\right.$, Acetone- $\left.d_{6}, \mathrm{TMS}, 25{ }^{\circ} \mathrm{C}\right) \delta 3.35(\mathrm{~s}, 3 \mathrm{H}$, O- $\left.\mathrm{CH}_{3}\right), 3.79\left(\mathrm{t}, 2 \mathrm{H}, \mathrm{N}-\mathrm{CH}_{2}-\mathrm{CH}_{2}-\mathrm{O}\right), 4.04\left(\mathrm{~s}, 3 \mathrm{H}, \mathrm{CH}_{3}-\mathrm{N}\right), 4.49$ (t, 2H, N-CH $\left.\mathrm{CH}_{2}-\mathrm{CH}_{2}-\mathrm{O}\right), 7.64$ (d, $2 \mathrm{H}, \mathrm{N}-\mathrm{CH}-\mathrm{CH}-\mathrm{N}), 8.92$ (s, $1 \mathrm{H}, \mathrm{N}-\mathrm{CH}-\mathrm{N})$; Anal. Calcd for $\mathrm{C}_{9} \mathrm{H}_{13} \mathrm{O}_{5} \mathrm{~N}_{3} \mathrm{~S}_{2} \mathrm{~F}_{6}: \mathrm{C}, 25.66 ; \mathrm{H}, 3.11 ; \mathrm{N}$, 9.97. Found: C, 25.39; H, 3.07; N, 10.21.

$\left[\mathrm{C}_{3} \mathrm{UC}_{4} \operatorname{mim}\right]\left[\mathrm{Tf}_{2} \mathrm{~N}\right]: 85.0 \%$ yield; ${ }^{1} \mathrm{H}$ NMR $\left(250 \mathrm{MHz}\right.$, Acetone- $\left.d_{6}, \mathrm{TMS}, 25{ }^{\circ} \mathrm{C}\right) \delta 0.92(\mathrm{t}, 3 \mathrm{H}$, $\left.\mathrm{NH}-\left(\mathrm{CH}_{2}\right)_{3}-\mathrm{CH}_{3}\right), 1.34\left(\mathrm{~m}, 2 \mathrm{H}, \mathrm{NH}-\left(\mathrm{CH}_{2}\right)_{2}-\mathrm{CH}_{2}-\mathrm{CH}_{3}\right), 1.42\left(\mathrm{~m}, 2 \mathrm{H}, \mathrm{NH}-\mathrm{CH}_{2}-\mathrm{CH}_{2}-\mathrm{C}_{2} \mathrm{H}_{5}\right), 2.05$ (m, $\left.2 \mathrm{H}, \mathrm{N}-\mathrm{CH}_{2}-\mathrm{CH}_{2}-\mathrm{CH}_{2}-\mathrm{NH}\right), 3.15$ (m, 2H, NH- $\left.\mathrm{CH}_{2}-\mathrm{C}_{3} \mathrm{H}_{7}\right), 3.19$ (m, 2H, N-CH $\left.-\mathrm{CH}_{2}-\mathrm{CH}_{2}-\mathrm{NH}\right)$, 4.03 (s, 3H, $\left.\mathrm{CH}_{3}-\mathrm{N}\right), 4.37$ (t, 2H, N-CH$\left.{ }_{2}-\mathrm{CH}_{2}-\mathrm{CH}_{2}-\mathrm{NH}\right)$ ), 5.65, 5.77 (t, 2H, NH-CO-NH), 7.67, 7.80 (s, 2H, N-CH-CH-N), 9.10 (s, $1 \mathrm{H}, \mathrm{N}-\mathrm{CH}-\mathrm{N})$; Anal. Calcd for $\mathrm{C}_{14} \mathrm{H}_{23} \mathrm{O}_{5} \mathrm{~N}_{5} \mathrm{~S}_{2} \mathrm{~F}_{6}: \mathrm{C}, 32.37 ; \mathrm{H}$, 4.46; N, 13.48. Found: C, 32.54; H, 4.43; N, 13.55. 